Canadian University Music Review

Revue de musique des universités canadiennes

\title{
Peut-on parler de narrativité en musique?
}

\section{Jean-Jacques Nattiez}

Volume 10, numéro 2, 1990

Alternative Musicologies

Les Musicologies Alternatives

URI : https://id.erudit.org/iderudit/1014886ar

DOI : https://doi.org/10.7202/1014886ar

Aller au sommaire du numéro

Éditeur(s)

Canadian University Music Society / Société de musique des universités canadiennes

ISSN

0710-0353 (imprimé)

2291-2436 (numérique)

Découvrir la revue

Citer cet article

Nattiez, J.-J. (1990). Peut-on parler de narrativité en musique? Canadian

University Music Review / Revue de musique des universités canadiennes, 10(2),

68-91. https://doi.org/10.7202/1014886ar

All Rights Reserved (C Canadian University Music Society / Société de musique des universités canadiennes, 1990
Ce document est protégé par la loi sur le droit d'auteur. L'utilisation des services d’Érudit (y compris la reproduction) est assujettie à sa politique d'utilisation que vous pouvez consulter en ligne.

https://apropos.erudit.org/fr/usagers/politique-dutilisation/ 


\title{
PEUT-ON PARLER DE NARRATIVITÉ EN MUSIQUE?
}

\author{
Jean-Jacques Nattiez
}

La question de la narrativité musicale, si elle n'est pas nouvelle, revient en force à l'ordre du jour de la réflexion musicologique. En mai 1988, une conférence sur le thème «Music and the Verbal Arts: Interactions» se tenait au Dartmouth College de Hanover, dans le New Hampshire. Quinze jours plus tard, un groupe de musicologues et de théoriciens de la littérature était invité aux Universités de Berkeley et de Stanford ${ }^{1}$ pour évaluer, au cours de quatre intenses tables rondes, si'il est légitime de reconnaître à la musique une dimension narrative. En novembre de la même année, le congrès annuel de l'American Musicological Society à Baltimore offrait une session intitulée «Text and Narrative», présidée par Carolyn Abbate, et, à l'initiative de Joseph Kerman, une séance consacrée à l'ouvrage d'Edward $\mathrm{T}$. Cone, The Composer's Voice (1974). Des articles abordent le sujet dans nos revues spécialisées: je pense tout particulièrement aux études de Anthony Newcomb parues dans 19th Century Music: "Once more 'between absolute and program music': Schumann's Second Symphony » (1984) et "Schumann and late Eighteenth-Century Narrative Strategies» (1987) dont il existe un équivalent français (1989) ou, du côté de la musicologie francophone, à l'article de Marta Grabocz paru en 1987: «La Sonate en si mineur de Liszt: une stratégie narrative complexe» et aux essais du sémiologue finlandais Eero Tarasti (1984 et 1989). A n'en pas douter, un bon nombre d'articles sortiront des colloques cités plus haut. Et on attend la parution du livre de Carolyn Abbate: Unsung Voices: Narrative in 19th Century Music.

Tout ce courant de réflexion tente de prendre au sérieux, de manière positive

1 Je remercie tout particulièrement Anthony Newcomb de m'avoir invité à ce symposium. Sans la liste de lectures fournie pour cette occasion et les échanges avec les autres participants, je n'aurais pas été en mesure de préparer le présent texte. J'ai moi-même soumis ce texte à Newcomb pour obtenir ses réactions aux critiques dont son approche fait ici l'objet et je le remercie pour l'accueil bienveillant et constructif qu'il leur a réservées. Ma reconnaissance s'adresse également à Carolyn Abbate, François Delalande et Jean Molino pour la pertinence de conseils. La présente version tient compte de leurs observations. 
ou critique, une intuition du sens commun: par l'oeuvre, le compositeur nous parle. Et a priori, nous pensons tous que Lévi-Strauss touche à quelque chose de vrai lorsque, à la fin de L'homme nu, il affirme: «Mythe codé en sons au lieu de mots, l'oeuvre musciale fournit une grille de déchiffrement, une matrice de rapports qui filtre et organise l'expérience vécue, se substitue à elle et procure l'illusion bienfaisante que des contradictions peuvent être surmontées et des difficultés résolues ... Il n'y a pas d'oeuvre musicale qui ne s'ouvre sur un problème et ne tende vers sa résolution, en donnant à ce terme un sens plus large mais conséquent avec celui qu'il a dans le langage musical» (1971: 587-8). Et à propos du Boléro de Ravel: "Comme un mythe, même une oeuvre dont la construction semble si transparente au premier coup d'oeil qu'elle n'appelle aucun commentaire, narre sur plusieurs plans simultanés une histoire en réalité fort complexe ${ }^{2}$, et à laquelle il lui faut donner un dénouement» (ibidem: 595).

Je ne cacherai pas que, jusqu'à ces dernières années, $j$ 'avais considéré la notion de récit ou de narration musicale comme une métaphore de plus à laquelle le langage humain doit recourir, avec ses pauvres moyens, pour tenter de cerner la spécificité du déroulement de la musique dans le temps. Mais à partir du moment où, dans le champ de l'analyse et du «musical criticism », un certain nombre d'excellents auteurs prennent cette métaphore au sérieux, l'esprit le plus rebelle aux approximations sémantiques se dit qu'il n'y a pas de fumée sans feu. Il accepte alors d'examiner pourquoi la notion de «récit musical » séduit les meilleurs esprits et d'évaluer s'il y a là la racine d'une orientation nouvelle pour l'analyse et la compréhension de la musique. Après tout, c'est parce que, au début des années soixante-dix, il m'a semblé que les modèles linguistiques pouvaient apporter quelque chose de fructueux à l'analyse musicale que, dans mes premiers travaux, j'ai comparé structures musicales et structures linguistiques ${ }^{3}$. La sémiologie peut être également tentée de confronter le déroulement musical au récit littéraire. Je vais essayer ici de clarifier mes vues à ce sujet.

Dans la mesure où la notion de récit est d'abord rattachée aux pratiques verbales, il semble nécessaire, dans un premier temps, de tenter de définir ce qu'est un récit littéraire. Comme on peut s'en douter, les définitions varient d'une théorie à une autre. Sans nécessairement adhérer à l'ensemble de ses

2 C'est moi qui souligne.

3 Cf. les numéros 5 et 10 de la revue Musique en Jeu (1971 et 1973) et nos Fondements d'une sémiologie de la musique (1975: deuxième partie). 
propositions, je prendrai comme point de départ les observations de Seymour Chatman dans son ouvrage désormais classique: Story and Discourse: Narrative Structure in Fiction and Film (1978). Selon lui, «il n'y a d'histoire que lorsqu'on recontre à la fois des événements et des existants ${ }^{4}$ » (1978: 113). Par exemple: «La marquise sortit à cinq heures». Il y a bien un «existant»: la marquise, et un «événement»: ce qu'elle fit en fin d'aprèsmidi. Et des relations s'instaurent entre «existants » et «événements»: «On ne peut pas rendre compte d'événements sans reconnaître l'existence de choses causant ces événements ou étant affectés par eux $»^{5}$ (ibidem: 34 ). Dans un récit, il y a à la fois une dimension linéaire - des événements se produisent à différents moments du temps - et des relations de cause à effet entre ces divers événements. Et Chatman précise quelque chose qui va se révéler important pour notre comparaison avec la musique: «La cause peut être explicite ou implicite ${ }^{6}$ (ibidem: 45 ). Si je lis dans un roman policier: «La marquise sortit à cinq heures. A cinq heures un quart, on trouva son mari mort dans le parc voisin», je suis tenté de créer une relation de causalité entre la sortie de la marquise et la mort de son mari, relation qui n'est que de l'ordre de l'hypothèse et qui crée précisément l'intérêt de l'intrigue.

De tout cela se dégagent deux niveaux d'existence du récit qu'il convient de distinguer pour le présent examen:

1) Quand je lis la phrase: «La marquise sortit à cinq heures", je n'ai pas besoin d'un titre pour comprendre que j'ai affaire à un récit. Au contraire, quand j'entends le début de l'Apprenti sorcier, il me faut savoir qu'il s'agit d'un poème symphonique pour aborder l'œuvre avec une intention d'écoute narrative. Des images peuvent venir à mon esprit, mais je dois aborder

4 «Stories only exist where both events and existents occur». Je n'adhère pas à la totalité du propos de Chatman car je ne suis pas certain que l' 'événement» et l'« existant » constituent les ingrédients minimaux d'un récit. Il écrit: «Il ne peut y avoir d'événements sans « existants ». Et bien qu'il soit vrai qu'on peut trouver dans un texte des «existants» sans événement (un portrait, un essai descriptif), il ne vientdrait à l'idée de personne de l'appeler un récit.» "There cannot be events without existents. And though it is true that a text can have existents without events (a portrait, a descriptive essay), no one would think of calling it a narrative » (1978: 113). Je n'en suis pas si sûr, car dans la description d'une personne ou d'un paysage, il y a une personne qui parle - l'écrivain - et qui, parmi l'infinité des choses qui peuvent être dites à propos de cette pesonne ou de ce paysage, a choisi. Pour nous, il reconstitue un monde et il nous raconte bien l'expérience qu'il en a.

5 «One cannot account for events without recognizing the existence of things causing or being affected by those events $»$.

6 «Causation may be explicit or implicit». 
l'oeuvre avec une stratégie d'écoute particulière pour construire un récit à partir d'elle. La démonstration est triviale: si la musique pouvait, par ellemême, être récit comme peut l'être le langage humain, elle nous parlerait directement et il n'y aurait plus de différence entre langage et musique.

2) Mais la narration n'est pas seulement inscrite dans la lettre du récit littéraire. Le texte est récit encore autrement: il a été imaginé par l'écrivain ou le conteur; il est re-construit par le lecteur. C'est précisément là que l'analogie peut exister entre récit littéraire et musique. Des titres comme l'Apprenti sorcier ou Du berceau jusqu'à la tombe, ou le récit autobiographique qui accompagne la Symphonie fantastique indiquent bien qu'il y a, de la part du compositeur, intention de suivre un enchaînement d'événements et de mettre en scène des «existants ». Mais c'est seulement si l'auditeur décide de relier la succession des événements sonores selon une intrigue, qu'il constitue l'oeuvre musicale en récit. Et c'est à la fois parce que le concept de récit est né avec la littérature, orale et écrite, et parce qu'il y a bien une différence ontologique entre récit littéraire et « récit» musical qu'on ne peut poser la question de la narrativité musicale sans prendre pour référence le récit littéraire.

On pourrait opposer à cette position de départ celle de Paul Ricoeur qui, dans Temps et récit, reconnaît à l'action humaine et aux événements historiques une dimension intrinsèquement sémantique. Il a thématisé cette manière de voir en analysant, dans un article très lu aux Etats-Unis, les similitudes entre l'action humaine et le texte (1971). Mais l'argumentation de Temps et récit mérite d'être regardée de près. Ricoeur reconnaît que «nous n'avons pas d'accès aux drames temporels de l'existence en dehors des histoires racontées à leur sujet par d'autres et par nous-mêmes » (1983: 113). Ce qu'il prête aux actions et aux événements, c'est ce qu'il appelle "une structure pré-narrative de l'expérience» (ibidem). "Sans quitter l'expérience quotidienne, ne sommes-nous pas inclinés à voir dans tel enchaînement d'épisodes de notre vie des histoires 'non (encore) racontées', des histoires qui demandent à être racontées, des histoires qui offrent des points d'ancrage au récit? » (ibidem). Les événements vécus constituent pour lui « une histoire à l'état naissant», une "narrativité inchoative» (ibidem), une «histoire potentielle»(1983: 114) ${ }^{7}$. Cette potentialité narrative de l'action humaine et de l'histoire est due, bien sûr, à l'inscription des événements dans le temps. Mais tout cela signifie bien que le récit proprement dit n'apparaît que lorsqu'une série temporelle d'objets et d'événements est prise en charge par un discours.

Comme pour Carolyn Abbate, nous croyons que le récit est non seulement

7 Tous les mots soulignés le sont par moi. 
une intrigue ou une histoire, mais aussi un acte, ce que Molino qualifie de conduite de récit ${ }^{8}$. On ne sera pas étonné que je retrouve ici la tripartition qui caractérise sa théorie sémiologique et que j'utilise dans mes travaux. Au moment où le romancier écrit l'histoire de la marquise, il sait si elle sera ou non la coupable. Mais la seule chose qu'il nous livre, c'est une trace à partir de laquelle le lecteur construit ses propres relations causales qui peuvent correspondre ou non à celles conçues par l'écrivain. Ce mécanisme est très évident dans le cas de la bande dessinée puisque, et c'est une partie de son charme, l'imagination du lecteur supplée au vide narratif qui existe entre deux images. C'est également celui que est à l'oeuvre lorsque nous entendons la musique avec, plus ou moins spontanément, une intention d'écoute narrative. Je discuterai l'existence de l'éventuelle narrativité musicale dans le cadre de cette conception tripartite pour tenter de cerner en quoi consiste l'impulsion narrative à laquelle la musique donne lieu.

Si tant de compositeurs ont choisi d'écrire des oeuvres musicales explicitement démarquées d'oeuvres littéraires, c'est sans doute parce qu'ils faisaient confiance aux possibilités sémantiques de la musique. En effet, à moins d'adopter une conception normativement formaliste de la musique comme le fit Hanslick, il est difficile de nier que des configurations purement sonores, indépendamment de toute suggestion textuelle, ont bien un pouvoir d'évocation. Les travaux de la psychologie expérimentale, de Francès (1958) à Imberty (1979 et 1981), sont là pour démontrer empiriquement que non seulement les auditeurs associent des images, des sentiments, des impressions à la musique, mais que, dans le pourcentage de réponses obtenues, il y a bien non pas unanimité mais convergence autour du fait vécu évoqué. On le sait aujourd'hui: la musique est capable de trois grands types de renvois extrinsèques, le spatio-temporel, le cinétique et l'affectif (Nattiez 1987: 155-164). Mais alors, où est-ce que le bât blesse ?

Le discours musical s'inscrit dans le temps. Il est fait de répétitions, de rappels, de préparations, d'attentes, de résolutions, et, au niveau de la syntaxe mélodique, c'est sans doute Leonard Meyer qui est allé le plus loin dans l'inventaire de ce qu'on pourrait appeler les techniques de la continuité. On est tenté de parler de récit musical à cause de l'existence de cette dimension syntaxique et temporelle de la musique.

8 «Qu'est-ce qu'un récit?», conférence inédite, présentée au département de littérature comparée de l'Université de Montréal le 6 mars 1975. 
Le récit littéraire, aussi, est fait d'attentes: «La marquise ...» Que fit-elle? Elle sortit. Elle sortit où ? On ne me le dit pas. Elle sortit quand? «À cinq heures». Pour quoi faire? Je le saurai plus tard. Mais ici, il n'y a pas d'ambiguïtés, en principe, sur l'identité des acteurs et la nature de leurs actions. La syntaxe linguistique, on le sait, repose principalement sur un sujet et un prédicat, et il n'y a pas de doute que le prédicat me dit ce qui est affirmé à propos du premier terme. Entre les deux, il y a une connexion logique.

Parfois sous différents termes, plusieurs théoriciens de la littérature ont distingué, dans le récit, entre l'histoire et le discours (par exemple Chatman 1978: 9). Cette dichotomie peut permettre de préciser la comparaison. Le contenu d'un récit, l'histoire qui est racontée, peut être «décollée» de son support linguistique pour être prise en charge par un autre médium, un autre type de discours, le film ou la bande dessinée. Il peut même être reconnaissable comme tel: dans les trois cas, on me raconte l'histoire de la marquise . . . La cassure avec le poème symphonique est plus nette: one ne peut pas le traduire. On peut résumer ou traduire le récit dont le compositeur s'est inspiré, ce qui est tout différent.

En musique, les connexions se situent au niveau du discours, pas au niveau de l'histoire. Que j'entende une marche dans la deuxième symphonie de Mahler, et j'imagine qu'il s'agit d'un groupe d'hommes mais je ne sais pas lesquels. La marche peut se rapprocher et s'éloigner, et même deux cortèges peuvent se croiser, comme dans Three Places in New England de Ives, mais je ne sais pas d'où ils viennent et où ils vont. En écoutant Till Eulenspiegel, et avec l'aide du titre, je peux bien admettre qu'il est question de la vie et de la mort d'un personnage. J'entends bien qu'il bouge, qu'il saute, etc . . . Mais que fait-il exactement ? Je ne le sais pas. "Music has no past tense», dit fort justement Carolyn Abbate. Elle peut évoquer le passé par des citations ou divers emprunts stylistiques. Mais elle ne peut raconter quelle action a eu lieu dans le temps (1990: chapitre 1). Le récit littéraire est invention, mensonge. La musique, elle, ne ment pas, car c'est nécessairement à moi, l'auditeur, que reviendra la charge de relier ces fantômes de personnages à des suggestions d'actions: il n'est pas dans les possibilités sémiologiques de la musique de relier un sujet et un prédicat. C'est pourquoi bon nombre d'approches «narratives » de la musique, si j'en crois ce que j'ai lu ou entendu ces derniers mois, me semblent céder à une illusion métaphorique: à quoi bon parler de narration schenkérienne, de métaphores et de métonymies musicales, quand, dans le discours de la musique, il n'est

9 Je laisserai de côté la question de savoir pourquoi l'adaptation cinématographique d'un roman connu ne restitue jamais vraiment tout ce que nous avions lu: je reconnais l'histoire, mais ce n'est pas vraiment le même récit. 
question que de jeux de formes et des réactions qu'elles suscitent? $\mathrm{Si}$, en écoutant la musique, je suis tenté par l'impulsion narrative, c'est bien parce que, au niveau du discours strictement musical, je reconnais des rappels, des attentes et des résolutions, mais je ne sais pas de quoi. Il me prend alors l'envie de compléter par des mots ce que la musique ne me dit pas parce qu'il n'est pas dans sa nature sémiologique de me le dire. Elle est, pour reprendre le mot paradoxal d'Adorno à propos de Mahler, «un récit qui ne raconte rien » (1976: 117).

C'est sans doute pour cela que le mot qui vient spontanément sous la plume d'auteurs qui reconnaissent à la musique une sorte de "narration en creux », est celui de geste. "Le geste mahlérien est celui de l'épopée», écrit Adrono (ibidem: 95), même s' «il reste interdit à la musique épique de décrire le monde qu'elle vise» (ibidem: 108). «Si la musique est vraiment un langage, écrit de son côté Cone, c'est un langage du geste: un langage des actions directes, des pauses, des démarrages et des arrêts, des montées et des chutes, de la tention et de la détente, des accentuations ... L'éconcé instrumental, privé de contenu verbal intrinsèque, va jusqu'à constituer ce qu'on pourrait appeler un medium de gestes purement symboliques ${ }^{10}$ (1974: 164). Dans cette perspective, cela ne me gênerait pas d'expliquer le succès universel du Boléro de Ravel, non pas par la simplicité de sa structure répétitive, mais par sa capacité à évoquer la montée irrésistible du désir jusqu'à l'éclatement orgastique des trombones.

Le livre de Cone, The Composer's Voice, n'a de sens que si l'on n'oublie pas que les concepts qu'il utilise, persona, agent, idea, sont, dans la musique instrumentale, "non spécifiés verbalement » et même "non identifiables » (1974: 94). "Les constituants de la musique - notes, accords, motifs n'ont normalement pas de référents ${ }^{11}$ écrit-il encore (1974: 161). Mais le risque reste grand de glisser de la métaphore narrative à une illusion ontologique: puisque la musique suggère le récit, elle serait elle-même récit. C'est cette nuance ténue, mais essentielle, qui fait toute la différence entre le récit littéraire et le «discours» musical.

Les faits historiques ne constituent pas, en eux-mêmes, un récit. Ils sont pris en charge par un récit qui leur donne sens. Comme l'a admirablement montré Paul Veyne (1971), je peux bien constater que des événements ont eu lieu

10 «If music is a language at all, it is a language of gesture: of direct actions, of pauses, or startings and stoppings, of rises and falls, of tenseness and slackness, of accentuations (. . .) Instrumental utterance, lacking intrinsic verbal content, goes so far as to constitute what might be called a medium of pure symbolic gesture ».

11 «Verbally unspecified», «unidentifiable». «The elements of music — notes, chords, motifs - normally have no referents ». 
- il y a eu une bataille, un traité a été conclu, des frontières ont été modifiées -, mais il n'y a interprétation historique proprement dite que lorsque je suis capable, en construisant une intrigue, d'établir entre les événements une relation de causalité qui les explique, c'est-à-dire en fait, de relier entre eux, par la logique d'un récit, les événements retenus. S'il en est ainsi, c'est parce que les événements sont comme des objets neutres inscrits dans le temps et qui, comme tels, sollicitent l'interprétation élaborée dans une narration.

Mais pas plus en histoire qu'en musique, ces objets neutres ne sont constitutifs, per se, d'un récit. On ne soulignera jamais assez que la narration construite à leur sujet occupe une position métalinguistique par rapport aux données ou aux événements que, pour reprendre la précieuse distinction de White (1981: 2), ils ne narrent pas mais narrativisent.

Leonard de Vinci avait déjà observé que «les taches sur un mur se mettent à vivre et à raconter des histoires ». Molino prolonge l'analyse: «Deux mots sur un mur font naître, non pas du tout une idée, un concept, une impression, ils font naître un récit ${ }^{12}$. Qu'il s'agisse de deux initiales dans un coeur, gravées sur un tronc d'arbre, le promeneur y lira la trace d'un roman d'amour qui nous dit à peu près: «Jean aime Marie», ou « Marie aime Jean », "Marie et Jean s'aiment», "Ici, Jean a recontré Marie», "Jean a retrouvé Marie », "Marie a retrouvé Jean», etc . . . La liberté d'interprétation reste grande, car ici le récit n'existe qu'à l'état virtuel, «que comme re-construction de celui à qui l'histoire est contée ou projection de celui qui la raconte ... Il est créé par celui qui perçoit les deux mots et qui établit entre eux, pour les relier, une histoire».

Tout comme les constituants d'une oeuvre musicale, les initiales gravées sur un mur ne constituent pas en elle-même un récit: elles sont le résultat d'une conduite de récit et elles en déclenchent une infinité d'autres chez les lecteurs. Deux conditions sont nécessaires et suffisantes pour cela. Il faut que, dans la trace,

1) un minimum de deux objets quelconques nous soit donné

2) et que ces deux objets s'inscrivent dans une dimension temporelle de sorte que l'on soit incité à établir une relation entre eux.

Pour illustrer ce mécanisme dans le domaine musical, je voudrais rapporter une expérience que j'ai menée il y a plusieurs années (Nattiez 1973). Elle a été effectuée auprès de trois cents élèves de classes de musique, à Montréal, de première et deuxième années de secondaire, et dont l'âge variait entre

12 Voir la note 8. 
onze et quatorze ans. On leur faisait entendre deux fois l'Apprenti sorcier de Paul Dukas sans leur indiquer le titre et il leur était dit: "Cette musique raconte une histoire. Laquelle ?» Puis ils ont eu quinze à vingt minutes pour écrire leur récit sous forme d'une petite narration. Certes, en écoutant le poème symphonique, il aurait été aussi étonnant que les élèves réinventent la ballade de Goethe qu'un singe, face à une machine à écrire, tape le Discours de la méthode de Descartes. L'objectif était de voir quel récit les élèves projetaient sur la musique.

Sur les trois cents réponses obtenues, quarante-sept ont été écartées: soit parce que l'élève n'a retenu qu'une impression générale sous forme d'une phrase, soit parce qu'il dit simplement qu'il aime ou qu'il n'aime pas l'oeuvre. Pour les besoins de l'analyse, les récits ont été ramenés à un résumé normalisé qui facilite les comparaisons. Par exemple:

«Dans la rue, une personne marche. Soudain arrivent des cavaliers. On entend un bruit de tambours. Il y a un combat. Les cavaliers repartent de plus en plus vite et on perçoit le bruit de leurs sabots. Puis c'est un arrêt. Repos. Il y a de la fumée de l'autre côté de la colline. Bataille: le sang coule, coule. Il y a beaucoup de morts, sauf un. Il retrouve son calme. Mais soudain quelque chose craque, et il se fait tuer ».

Ou encore:

«La nuit, dans un magasin de jouets. Tout est tranquille. Soudain, un jouet s'éveille. Un soldat de plomb fait la cour à une poupée. Mais une marionnette arrive, et c'est une lutte sans fin. Le soldat et la poupée s'en vont au loin, mais ils sont poursuivis. Ils courent de plus belle et se débarrassent de l'assaillante. A l'aube, le marchand revient et retrouve les objets à leur place ».

Enfin:

«Un homme marche tranquillement sur la route, lorsque surviennent des chiens. Sa marche tranquille continue, puis il se met à courir jusqu'à un arbre. Les chiens s'éloignent et l'homme descend. Il repart. Il est de nouveau surpris par les chiens, il se réfugie dans une vieille baraque. Il est pursuivi jusque chez lui. Les chiens s'en vont».

La lecture de l'ensemble des réponses fait apparaître le conditionnement culturel des récits. Etant entendu qu'un même récit a pu être placé dans plus d'une des catégories qui suivent, on relève:

103 récits d'aventure (voyage d'un jeune garçon, escalade en montagne, etc.)

36 récits de guerre (révolutions, conflits armés)

28 histoires d'animaux

26 histoires de chevalerie et du Moyen-âge (princes et princesses)

21 récits «à suspense» (espionnage, policier, vol) 
17 histoires sentimentales

14 récits biographiques (de la naissance à la mort, la vie de Beethoven (!), etc.)

13 évocations de musique pure (le récit décrit les instruments ou imagine qu'on est au concert)

6 récits fantastiques

4 évocations de danses et ballets.

L'examen des deux cent cinquante-trois réponses conduit aux constatations suivantes:

1) Il permet de savoir à quelles sections l'élève a particulièrement fait un sort. Le récit construit est un indice de l'analyse formelle intuitive que l'élève a faite du poème symphonique par le biais de sa verbalisation narrative. Ainsi, au travers des récits, on distingue:

— une section initiale, jusqu'à l'arrivée de l'apprenti : 179 réponses;

- une section de transition, de cette arrivée au début (non compris) de la marche du balai: 60 réponses;

- une section centrale, constituée de deux crescendos: 232. Sur ces deux cent trente-deux réponses, vingt-quatre ont remarqué les deux moments de la section.

— une «dégringolade» qui est censée traduire la «mort» du balai: 56 réponses;

- une période de calme avant la reprise du thème du balai par les deux bassons: 41 réponses;

- la reprise de la section centrale avec les deux bassons: 138 réponses;

- la mort des deux balais après l'intervention du maitre sorcier: 18 réponses;

- la reprise de la section intiale: 175 réponses;

- l'accord final: 49 réponses.

Dans le détail, on remarque:

1. que la césure entre les deux moments de la section centrale est beaucoup moins bien identifée que ces sections elles-mêmes;

2. que si les sections initiale et finale ont retenu l'attention dans la même proportion, neuf réponses seulement ont établi explicitement un rapport d'identité entre elles;

3. que, dans l'ensemble, l'identification thématique est faible: les récits ne semblent pas attribuer à un personnage un thème caractéristique: les «formules magiques» des trombones ne sont pas relevées de façon spécifique, alors que les élèves sont sensibles aux «chutes», surtout après la première partie de la section centrale;

4. que l'accord final qui, d'après l'argument, ne semble pas avoir de signification particulière, fait l'objet d'une interprétation sémantique caractérisé dans un nombre respectable de cas, sans doute à cause de sa 
position et du contraste avec la reprise de la section calme du début.

2) L'enquête démontre surtout que, étant invités à une écoute narrative, les élèves rendent cohérents après coup la succession des événements sonores en imaginant des relations sujet-prédicat que, par elle-même, la musique ne peut expliciter. Au-delà du détail des récits obtenus, et dans la mesure où quatre-cinquièmes des réponses ne retiennent que la section initiale, un événement central (qui néglige la cassure du balai en deux) et la section finale, c'est le pattern "calme/poursuite/calme» qui se dégage de l'expérience. Il s'explique, comme le lassaient attendre les analyses de Francès, par des aspects cinétiques et dynamiques de la substance musicale. Au-delà de toute narration construite après coup, cette constante paradigmatique des réponses obtenues nous restitue, en termes sémantiques, une image de l'effet produit par la musique sur les auditeurs.

Ces observations sembleraient apporter une justification expérimentale à l'approche narrative des oeuvres de musique instrumentale de Schumann par Anthony Newcomb: en écoutant une oeuvre, nous y reconnaissons l'évocation d'actions, de tensions et de dynamismes analogues à ceux que véhicule l'oeuvre littéraire. Mais devant ses belles études, je suis à la fois admiratif et critique. Admiratif parce que, stimulé par les recherches narratologiques, Newcomb essaie de rendre compte d'un niveau de l'organisation musicale qui n'est plus celui de l'organisation syntaxique et formelle, mais celui d'éléments fonctionnels qui articulent l'oeuvre à un niveau plus élevé. Critique, parce que je ne suis pas certain, comme il l'affirme, que l'enchaînement de ces éléments fonctionnels soit à proprement parler constitutif d'un récit.

Son idée de base, c'est que ce niveau qualifié par lui de narratif réponde, dans plusieurs pièces d'un ensemble historiquement délimité, à un "archétype d'intrigue», "a plot archetype». Celui de la $V^{\mathrm{e}}$ et de la $\mathrm{IX}^{\mathrm{e}}$ de Beethoven, tout comme celui de la II $^{\mathrm{e}}$ Symphonie de Schumann correspondrait à la succession «narrative » de deux états affectifs: «souffrance, suivi de guérison ou de rédemption» («suffering, followed by healing or redemption»). Cette approche permettrait donc de saisir un nouveau niveau de signification: «La conception de la musique comme roman composé, comme une vraie suite psychologique d'idées, a été et reste une voie importante pour la compréhension d'une grande partie de la musique du $\mathrm{XIX}^{\mathrm{e}}$ siècle: la Cinquième Symphonie de Beethoven, par exemple, a été ainsi comprise dès le début par au moins quelques auditeurs. Aussi, nous pouvons trouver à la base de quelques symphonies un schéma évolutif d'états mentaux de la même façon que les formalistes russes et les structuralistes ont trouvé, 
à la base des romans et des contes, un parmi plusieurs archétypes d'intrigue $»^{13}$ (Newcomb $1984:$ 234).

Or si notre expérience à propos de Dukas semble bien confirmer qu'au-delà de la projection sur l'oeuvre d'une intrigue narrative explicite, les auditeurs perçoivent ce que Newcomb appelle tout à fait pertinemment des « functional events » (1987: 165), je ne suis pas certain qu'il soit légitime pour autant de parler de récit.

La constante paradigmatique «calme/poursuite/calme», en effet, est induite des récits réels construits par les auditeurs. Elle nous permet de savoir quels aspects de la grande forme, sémantiquement traduite, ont été perçus. Mais précisément, elle ne nous raconte pas qui est calme et pourquoi, qui poursuit qui, et pourquoi il y a retour au calme. Comme y insistent Abbate et White (1981: 9-11), l'auditeur comme l'historien et, au deuxième niveau que nous avons distingué au début, le lecteur, sont des «gap-fillers», des «boucheurs de trous », des «combleurs de lacune» (si, en français, on peut s'exprimer ainsi). Le récit à proprement parler n'est pas dans la musique, mais dans l'intrigue imaginée et construite par les auditeurs à partir d'objets functionnels.

Pour traiter de la narrativité musicale, Newcomb compare sa démarche à celle de la narratologie structuraliste ${ }^{14}$. Or si l'on se reporte à la Morphologie du conte de Propp (1970), on constate que les divers moments du conte merveilleux sont résumés selon une succession d'actions canoniques: «Un des membres de la famille s'éloigne de la maison», "Une interdiction est imposée au héros», "L'interdiction est transgressée », etc . . . Propp ramène cette chaîne de formules à une suite de fonctions exprimées par un seul mot: «éloignement », « interdiction », «transgression », « interrogation », etc . . . Elles semblent comparables à notre «calme/poursuite/calme» de l'Apparenti sorcier à l'interprétation des symphonies de Beethoven et

13 «The conception of music as composed novel, as a pyschologically true course of ideas, was and is an important avenue to the understanding of much nineteenth century music: Beethoven's Fifth Symphony, for example, was so understood by at least some listeners from the outset. Thus we may find at the basis of some symphonies an evolving pattern of mental states, much as the Russian formalists and the structuralists find one of several plot archetypes at the basis of novels and tales".

14 Cf. aussi son texte français (1989: 13-15). Dans une communication personnelle, Newcomb nous indique qu'il considère aujourd'hui la référence à Propp comme erronée, mais dans la mesure où sa conception de la narrativité musicale repose toujours sur la notion de "plot archetype», il faudra attendre les prochains développements des ses recherches pour savoir exactement quel statut épistémologique il accorde à ces deux notions. 
Schumann citées plus haut. Mais alors que les fonctions dégagées par Propp montrent quels sont les axes paradigmatiques communs à des récits relevant d'un même genre, alors que notre constante paradigmatique nous renseigne sur un fait de perception, la transposition de Newcomb nous dit en réalité que, pour l'auditeur, toute oeuvre instrumentale «narrative » n'est pas en elle-même un récit, mais l'analyse structurale en musique d'un récit absent.

Qui plus est, si, dans la séquence "souffrance-rédemption», on reconnaît sans peine un schéma romantique, il n'est en rien comparable à la chaîne des fonctions identifiées par Propp dans un genre rigoureusement délimité, le conte merveilleux, où ces fonctions peuvent connaître des effacements, des substitutions, des permutations. Le couple « souffrance-rédemption » constitue une caractérisation tellement générale qu'on se demande bien si nous sommes en présence, à proprement parler, d'une structure narrative ou s'il ne s'agit pas plutôt d'un trait culturel qui déborde la littérature, de la même façon que, chez les Japonais, la succession «attente + action rapide et violente » est moins une structure littéraire qu'un schéma typique de comportement, un schème culturel et une manière d'être. Or, un schéma de comportement, c'est quelque chose de bien différent et de plus large, de plus vague aussi, qu'une structure narrative.

Si j'insiste sur cette différence, c'est parce que le détour par la métaphore narrative risque d'occulter une dimension fondamentale du fonctionnement sémiologique de la musique. Je ne nie absolument pas que des moments particuliers des symphonies de Beethoven connotent les idées de "souffrance » et de "rédemption», que l'enchaînement des tonalités dans la $I^{e}$ de Beethoven obéisse à une logique dramatique plutôt que formaliste, comme l'a bien montré Leo Treitler (1982). Ce que je dis, c'est que le lien narratif entre ces moments de la musique ou ces tonalités n'est pas et ne peut pas être inscrit dans la syntaxe musicale, alors que le lien entre «la marquise » et «sortit à cinq heures», lui, est bien inscrit dans la syntaxe de la phrase. Je partage entièrement la position de Newcomb et de Treitler selon laquelle la signification de ces enchaînements ne peut être reconstituée que par les voies de l'herméneutique historique. Simplement, je serais sans doute davantage enclin à chercher à distinguer - lorsque les données le permettent -

1) ce que l'intention narrative a pu être pour Beethoven,

2) comment elle a été comprise par les contemporains, (en particulier chez ces auditeurs spécialisés que sont les critiques),

3) comment cette même succession est comprise aujourd'hui,

4) quels sont les différents modèles, pour le compositeur et les diverses catégories d'auditeurs, de ces constructions narratives. Si la littérature peut être un de ces modèles, et à des niveaux différents comme j'essaierai de le montrer dans la suite de cet article, elle n'est certainement pas le seul: la poésie, la philosophie, les beaux-arts peuvent intervenir, pas nécessairement 
comme porteurs de schémas narratifs structuraux, mais comme réservoir de traits philosophiques, idéologiques et culturels propres à une époque.

En un mot, je ne pense pas que ce soit en ramenant la signification des oeuvres instrumentales au modèle réducteur de l'analyse structurale des récits qu'on réussira à cerner ce qui fait la spécificité de ce niveau de signification à une époque donnée, et que Newcomb a eu tout à fait raison de dégager, lorsqu'il est pris en charge par cette forme symbolique particulière qu'est la musique. C'est toute la question des médiations entre les formes symboliques et les traits qui contribuent à l'esprit d'une époque que, d'un point de vue sémiologique, il me semble nécessaire de poser.

Il n'est évidemment pas possible, dans le cadre de cet article, de présenter une théorie complète de ces rapports. Par contre, et en «redescendant » au niveau de relations plus immédiates entre musique et récit, je voudrais évoquer quelques cas empiriques de médiations.

Toute perception de la musique déclenche l'établissement d'un lien entre l'oeuvre et l'expérience vécue de l'auditeur. Si on a le sentiment que la musique raconte une histoire qui ne nous est pas dite, c'est peut-être parce que, sémantiquement parlant, la musique est capable de diverses formes d'imitation, et que, parmi elles, il lui est possible d'imiter l'allure d'un récit littéraire.

Cela fait longtemps que les linguistes se sont penchés sur ce qu'ils ont appelé les «éléments musicaux » du langage humain. Musique et langage ont en commun d'être constitués d'objets sonores. Dans le langage comme en musique, il y a des rythmes et des accents, des longueurs de notes et de syllabes, et ce n'est pas un hasard si, en grec, mousikê désignait la poésie lyrique, c'est-à-dire quelque chose qui était à la fois ce que nous appelons aujourd'hui musique et poésie; s'il n'est pas impossible d'expliquer la dimension rythmique et métrique de la poésie lyrique moderne par ses liens indissociables, dans ses origines, avec la musique (Molino-Tamine 1987). Mais il y a plus.

Dans le langage, à l'exception des langues à ton, les voyelles n'ont pas de hauteurs fixes, mais l'enchaînement des syllabes crée des courbes intonatives - les prosodèmes - auxquelles certains phonéticiens - je pense en particulier à Pierre Delattre (1966, 1967 et 1969) — ont consacré des études spécialisées. Ceci est décisif: musique et langage partagent la linéarité du discours et l'utilisation d'objets sonores. La musique est capable d'imiter la courbe intonative d'un récit. 
Dans le $X V^{e}$ quatuor de Beethoven, il n'est pas nécessaire d'avoir lu le motto en tête du dernier mouvement, "Muss es sein ? Es muss sein", pour reconnaître dès le début que nous sommes en présence d'une question, suggérée par la quarte ascendante, suivie d'une réponse double, évoquée par les quartes descendantes. Et à partir de là, le reste du mouvement peut être interprété comme la transposition musicale d'un dialogue. Nous ne savons pas ce qui est dit, mais Beethoven nous dépeint le caractère de l'échange, un peu comme si nous en captions les inflexions à travers un mur ou que nous écoutions une conversation dans une langue que nous ne connaissons pas.

Les voix du quatuor ont donc bien ici le caractère de ce que Cone, à propos du dialogue du hautbois et du cor anglais dans la «Scène aux champs » de la Symphonie fantastique, appelle des "personnages virtuels» ou, plus précisément dans sa terminologie, des «agents virtuels» (1974: 88). C'est évidemment le mot «virtuel» qui me semble ici le plus important. Sans doute est-ce pour la même raison que le vocabulaire traditionnel de l'analyse de la fugue utilise des termes comme "sujet», "réponse», "exposition", "discussion» et "sommaire». "Il suggère», dit Cone, "le modèle d'une conversation sur un sujet donné $»^{15}$ (ibidem: 113). Pour reprendre la belle expression de Abbate (1990), la musique nous fait entendre «le son de la voix qui raconte ${ }^{16}$ mais nous ne savons pas exactement de quoi parle cette voix.

Ces cas d'imitation de l'intonation ne sont pas uniques. Nettl (1956: 2) a suggéré que l'accentuation de la première syllabe en tchèque explique le schéma accentuel de la phrase musicale des compositeurs tchèques, et je connais au moins deux recherches (Hall 1953 et Callaghan 1985) qui ont cherché à retrouver les intonations de l'anglais dans la musique de Elgar. Hall écrit : «En anglais britannique comme en anglais américain, la fin d'une phrase déclarative est caractérisée par une intonation descendante, d'un ton relativement haut à un ton relativement bas. C'est également ce qui se produit dans une question commençant par un interrogatif (par ex., Where are you going?). Mais dans les questions qui ne commencent pas par un interrogatif (par ex., Are you coming ?), l'anglais américain et la plupart des langues européennes utilisent une intonation ascendante marquée, alors que l'anglais britannique a recours à la même intonation descendante que dans Where are you going ?» Se tournant vers la musique de Elgar, Hall rapporte que chez lui - ce qu'il faudrait évidemment vérifier statistiquement - «un grand nombre de ses thèmes présente une tendance descendante prédominante; pensez par exemple», dit-il, "aux principaux motifs de Falstaff, au thème initial de l'Introduction and Allegro, au premier sujet de la seconde

15 «It suggests the model of a conversation on an announced topic ».

16 « The sound of voice of narrating ». 
symphonie, et à de nombreux autres». «Pas étonnant, conclut-il plus loin, «que les Anglais aient le sentiment qu'il y a chez Elgar quelque chose de particulier qui leur est propre, et que les non-britanniques ne peuvent apprécier ${ }^{17}$ (Hall 1953: 6). Si nous avons bien ici une explication possible de l'analogie entre musique et allure du discours, l'analyse nous rappelle également qu'elle peut être restreinte à une culture particulière. Par un processus spécifique, une langue a laissé sa marque dans une musique.

On retrouve une idée analogue chez d'autres chercheurs. Dans une vaste fresque qui doit plus à l'imaginaire personnel de Lévi-Strauss qu'à une patiente investigation scientifique, l'ethnologue a suggéré que, dans l'histoire des formes symboliques, la musique a pris la place du mythe dont elle aurait épousé les structures: "Il semble bien que le moment où musique et mythologie ont commencé d'apparaître comme des images retournées l'une de l'autre, coïncide avec l'invention de la fugue . . . Tout se passe comme si la musique et la littérature s'étaient partagé l'héritage du mythe. En devenant moderne avec Frescobaldi puis Bach, la musique a recueilli sa forme, tandis que le roman, né à peu près en même temps, s'emparait des résidus déformalisés du mythe, et désormais émancipé des servitudes de la symétrie, trouvait le moyen de se produire comme récit libre» (1971: 583). Si l'on peut éprouver quelques réserves à l'égard de cette vision, l'hypothèse de Lévi-Strauss repose sur une conception des rapports entre musique et langage qui n'est pas sans intérêt: «En se séparant [du langage], la musique a conservé l'empreinte en creux de sa structure formelle et de sa fonction sémiotique: il ne saurait y avoir de musique sans langage qui lui préexiste et dont elle continue de dépendre, si l'on peut dire, comme une appartenance privative» (ibidem: 579). Il y a là, en effet, une intuition profonde que la musicologie allemande avait explorée dès 1947 (Georgiades) et que la musicologie américaine a récemment reprise (Norton 1984), sans, apparemment, qu'il y ait eu la moindre connexion entre les trois chercheurs.

Utilisant le seul texte dont on sait qu'il est resté inchangé tout au long de l'histoire de la musique, Georgiades montrait en effet comment chaque

17 «A falling pitch, from relatively high to relatively low, characterizes the end of a declarative sentence in both British and American English, and also a question beginning with an interrogative word, e.g. Where are you going? But in questions not beginning with an interrogative (e.g. Are you coming ?) American English and most European languages use a sharply rising intonation, whereas British English has the some falling pitch that it has in Where are you going?» ... « A great many of his themes show a predominantly falling trend; think, for instance, of the main motives of Falstaff, the introductory theme of the Introduction and Allegro, the first subject of the Second Symphony, and a host of others, » . . « No wonder that the English felt there is something peculiarly 'all their own' about Elgar, which the non-English fail to appreciate ». 
époque a traité les paroles cononiques de la Messe, et comment, par comparaison, le style de chaque pays a été influencé par les structures, notamment rythmiques et accentuelles, de la langue correspondante. Mais surtout, il faisait la démonstration que les musiques instrumentales, en s'émancipant progressivement de la musique vocale dominante jusqu'au baroque, ont conservé la trace des langues avec lesquelles elles avaient été en contact pendent au moins dix siècles. Norton a évoqué brièvement une hypothèse analogue sous le terme évocateur de "linguistic preformation » (1984: 65-71) qu'il développe surtout lorsqu'il tente de démontrer que la structure de la forme-sonate est, selon lui, analogue aux différentes moments de la dispositio rhétorique que Mattheson (1739) avait reprise à son compte. Ainsi, à l'exordium correspond l'introduction, pas toujours présente; à la narratio et la divisio, l'exposition; à la confirmatio et la confutatio, le développement; à la peroratio, la récapitulation et la coda (Norton 1984: 202-5).

Dans cette perspective, la musique n'est pas, à proprement parler, langage ou récit mais une forme symbolique spécifique qui a, parmi d'autres possibilités, la capacité sémiologique d'imiter l'allure d'un récit, d'un style ou d'un mode narratif, ce que Abbate (1990), précisément, dénomme «a mode of narrating ».

S'il en est ainsi, il est légitime de se tourner maintenant du côté des stratégies compositionnelles et de voir comment le compositeur peut tirer partie de la narrativité littéraire.

J'en ai fait l'expérience directe lorsque le compositeur québécois Denys Bouliane m'a fait entendre son oeuvre symphonique: Le cactus rieur et la demoiselle qui souffrait d'une soif insatiable. En entendant cette pièce faite d'une succession de développements à chaque fois interrompus, je songeais immédiatement au roman d'Italo Calvino, Si par une nuit d'hiver en voyageur, que j'avais lu quelque temps auparavant. J'en fis part au compositeur, qui me confirma sur le champ qu'il s'était effectivement inspiré du style narratif (et non du contenu) de cet ouvrage.

Cet exemple contemporain démontre, au cas où l'on en douterait, qu'il est possible à un compositeur de s'inspirer musicalement d'un style littéraire. Et je n'ai pour ma part aucune peine à suivre Newcomb lorsqu'il suggère que Schumann a pu tirer certaines de ses idées relatives à la forme musicale de la forme des romans de Jean-Paul (1984: 240). Les témoignages qu'il cite de critiques de l'époque et les propos de Schumann lui-même, légitiment pleinement son analyse. Ces mêmes éléments, révélateurs du Zeitgeist, rendent tout à fait crédible l'idée que, dans le Carnaval, Schumann a été 
influencé par la technique du Witz, «à savoir la faculté de découvrir des liens subtils sous une surface d'apparence incohérente ou d'extrême discontinuité » (1989: 18). Avec les «lettres dansantes», Schumann utilise «une simple petite cellule de hauteurs pour construire des mélodies qui, à la surface, diffèrent dans leur rythme, leur contour mélodique global, leur caractère, leur tempo " (ibidem $)^{18}$, la cellule assurant le lien subliminal entre les fragments constitutifs de l'oeuvre.

Où j'ai davantage de difficultés à suivre Newcomb, c'est lorsqu'il qualifie de "narrative game» le fait que Schumann joue avec les habitudes formelles établies à la fin du XVIII ${ }^{\mathrm{e}}$ siècle et au début du XIX ${ }^{\mathrm{e}}$, en particulier par le quatuor beethovénien. L'analyse qu'il propose du dernier mouvement du Quatuor à cordes op. 41, no. 3 est parfaitement convaincante du point de vue musical: «Dans le refrain aussi bien que dans le mouvement dans son ensemble, les fonctions des événements successifs finissent par ne pas être ce dont elles avaient l'air lorsqu'on les avait rencontrées pour la première fois, ni ce qu'elles devaient être selon l'intrigue paradigmatique » (ibidem: 20$)^{19}$. Mais parce qu'il existe une convention paradigmatique des successions et transformations à l'oeuvre dans un rondo, a-t-on pour autant le droit de parler de « fil du récit» schumannien, de «conventions narratives du rondo » ${ }^{20}$ ?

La question n'est pas académique, car si nous voulons comprendre non seulement la spécificité de la musique comme forme symbolique mais aussi les relations possibles entre déroulement musical et récit littéraire, il importe de distinguer clairement:

- d'une part, le fait, bien légitime dans le cadre d'une poïétique, qu'un compositeur crée à partir d'une sorte de "matrice conventionnelle», d'un modèle virtuel établi par la pratique compositionnelle courante à une époque que le novateur bouscule et dépasse jusqu'à ce qu'il crée, par son influence, une nouvelle norme;

- et d'autre part, l'idée qu'une novation formelle ait pu apparaître sous l'influence d'un mode de narration littéraire, le Witz dans le cas précis du Carnaval.

18 « The faculty by which subtle underlying connections are discovered in a surface of apparent incoherence, of extreme discontinuity» . . « A single little cell of pitches was used to build up melodies that were superficially different in rhythm, overall melodic contour, character, tempo, and so on ». J'utilise la traduction française de François Rüegg parue dans Contrechamps. Je dois à Etienne Darbellay la communication de l'original anglais de cet article.

19 «In both refrain and movement as a whole the functions of the successive events turn out not to be what they seemed to be when first encountered, and not what they should be according to the paradigmatic plot».

20 «The narrative thread», «the narrative conventions of the rondo». 
Dans son travail, Newcomb ne confond pas ces deux facteurs poḯtiques, mais en qualifiant le premier de «jeu narratif», il risque de diluer la spécificité de l'influence de la littérature sur Schumann dans une analyse musicale aussi éclairante que stimulante, mais abusivement et métaphoriquement qualifiée de narrative.

A ces deux facteurs, j'en ajouterai un troisième. Le fragment 392 de l'Athenaeum dit: «Beaucoup de compositions musicales ne sont que des traductions de poèmes dans la langue de la musique». Mais le sont-elles en suivant strictement la structure narrative du récit littéraire ? N'y a-t-il pas possibilité, pour la musique, de prendre en charge une idée générale véhiculée par un texte?

Si, dans le cas du Carnaval, Newcomb a établi de façon convaincante le lien avec l'esprit du Witz, il est possible de montrer qu'il s'est inspiré aussi d'un thème spécifique des Flegeljahre de Jean-Paul.

La recherche musicologique, biographique et historique a élucidé bien entendu la signification personnelle des allusions à Asch, la ville natale de la fiancée Ernestine; nous savons quels personnages de Jean-Paul - Walt et Vult - se cachent derrière Eusébius et Florestan, qu'à Chiarina correspond Clara et à Estrella, Ernestine, etc . . . Mais pourquoi deux compositeurs dans cette farandole de masques? On se contente généralement d'y voir un hommage à Chopin et Paganini, ce qui est sans doute vrai si l'on se réfère aux critiques de Schumann. Mais n'y aurait-il pas davantage?

Papillons fut composé deux ans avant Carnaval, et nous savons que Schumann a fait correspondre les dix premières pièces de cette oeuvre à dix paragraphes du chapitre 63 des Flegeljahre de Jean-Paul, le bal de masques (Boetticher 1941: 611-3). Par ailleurs, Jacques Chailley (1971) a montré qu'il existait un nombre suffisant d'analogies entre le texte de Jean-Paul et le contenu manifeste du Carnaval pour que l'on puisse affirmer que ce même chapitre est aussi une des sources d'inspiration de ces pièces pour piano. Il prend alors l'envie de relire ce texte et de voir s'il n'explique pas de manière plus organique la présence des compositeurs. La mise en musique du bal masqué semble alors répondre à un projet esthétique plus profond.

Jean-Paul écrit ceci: «Un bal en masques est peut être la réalité la plus sublime que la vie puisse inventer à l'imitation du jeu poétique. Devant le poète, toutes les classes et tous les Temps sont à égalité; toute apparence n'est que vêtement, l'intérieur toute joie et sons. Ainsi les hommes composent ici la figure poétique d'eux-mêmes et de la vie ... Tout est loué dans un seul cercle aérien et joyeux qu'anime un mouvement magnifique, on pourrait dire 'prosodique' -, la musique, pays des âmes au même titre 
que les masques sont le pays des corps. $\gg^{21}$

Ce passage du roman est important parce qu'il compare l'objet de la description romanesque avec l'art poétique lui-même et renverse la perspective habituelle: dans un bal masqué, la vie est imitation de l'art. Et ici, cette imitation fait appel à deux moyens: les vêtements et les masques sont la figuration poétique des corps, la musique, la figuration des âmes. En mettant en musique le Bal masqué de Jean-Paul, Schumann vient compléter l'effort du romancier pour montrer, comme le dit un de ses personages, comment «l'histoire entière de l'humanité ne paraît qu'un long bal travesti ». Avec les mots, Jean-Paul ne pouvait que décrire le bal; avec la musique, Schumann nous fait entendre la voix des âmes cachées derrière les masques.

Dans une lettre à sa famille du 17 avril 1832, Schumann s'inquiétait de savoir si, après avoir lu le dernier chapitre des Flegeljahre, «quelque chose de l'amour angélique de Wina, de la nature poétique de Walt et de l'âme lumineusement pénétrante de Vult se reflète correctement dans les Papillons » (cité dans Lippman 1964: 318). Il n'y a pas de raison de penser que Carnaval, dont la grande majorité des pièces porte le nom d'un personnage, ne répond pas au même objectif. Dans son ample étude sur l'esthétique de Schumann, Lippman a souligné l'importance du concept d'âme chez le compositeur (ibidem: 319), et c'est précisément ce que, selon Jean-Paul, la musique a la tâche de traduire dans un bal masqué ...

Dans sa pratique de critique, la description poétique schumanienne, miroir de l'effet que l'oeuvre produit sur nous, était posée comme un équivalent de la musique (ibidem: 323 ). Dans sa pratique de compositeur, les sons font entendre l'âme des personnages romanesque. On comprend mieux alors pourquoi les compositeurs doivent figurer dans cette galerie de portraits. Resitué par rapport aux propos du romancier, le Carnaval qui s'achève par la marche des Davidsbündler contre les Philistins est une sorte de manifeste esthétique, une prise de position de Schumann sur les rapports de complémentarité entre musique et littérature: non seulement sa structure s'inspire-t-elle du Witz littérature, mais son propos est de faire entendre les âmes dont la musique est le pays et que seul le compositeur, à la différence du romancier et parce que la musique est un art transcendental, a la capacité d'évoquer.

21 «Ein Ball en masque ist vielleicht das Höchste, was der spielenden Poesie das Leben nachzuspielen vermag. Wie vor dem Dichter all Stande und Zeiten gleich sind und alles Äussere nur Kleid ist, alles Innere aber Lust und Klang, so dichten hier die Menschen sich selber und das Leben nach . . . Alles Feindliche und Freundliche wird in einer leichten frohen Kreis gerundet, und der Kreis wird herrlich wie nach dem Silbenmass bewegt, nämlich in der Musik, diesem Lande der Seelen, wie die Masken das Land der Körper sind». Traduction Beaufils in Chailley (1971: 31). 
Cette hypothèse interprétative, je l'ai développée à partir d'un passage des Flegeljahre. Elle semble confirmer une des conclusions majeures de Lippman: «C'est bien la littérature qui apparaît comme la force formatrice de base qui affecte aussi bien sa musique, ses critiques et sa théorie esthétique $»^{22}$ (ibidem: 342 ). Mais en proposant cette herméneutique du côté de la poétique, je suis bien conscient que j'ai débordé de point de départ que m'avait fourni Newcomb en démontrant l'analogie entre les structures du Carnaval et la technique du Witz. Je n'ai pas cherché dans les Flegeljahre un modèle narratif spécifique dont le compositeur proposerait un équivalent musical, mais tenté de montrer que forme et contenu (puisqu'ici, l'oeuvre fait clairement allusion à des personnage) découlent ensemble d'un projet esthétique étroitement lié à l'enseignement de Jean-Paul romancier. Je n'affirme pas que, dans Carnaval, Schumann nous raconte, note après note, pièce après pièce, que la musique est le pays des âmes. Je suggère seulement que, par l'ensemble de cette oeuvre, c'est là ce qu'il veut nous dire, même si, pour le comprendre, il est nécessaire, au-delà de l'audition et grâce à l'herméneutique historique, de pénétrer les arcanes de son processus créateur.

On sera peut-être déçu que je sois parvenu au terme de cette investigation à des conclusions aussi négatives.

J'ai tenté de montrer, en effet, qu'en elle-même, et à la différence de bien des énoncés linguistiques, la musique n'est pas un récit et que toute description de ses structures formelles en terme de narrativité n'est qu'une métaphore superflue. Mais si on a été tenté de le faire, c'est parce que la musique partage avec le récit littéraire le fait qu'en elle, des objets se succèdent: cette linéarité est alors incitation à une conduite de récit qui narrativise la musique. Comme elle possède certaines capacités d'évocation imitative, il lui est possible de mimer l'allure d'une narration sans que nous sachions jamais quel est le contenu de ce discours, et cette influence des modes narratifs peut contribuer à la transformation des formes musicales. Mais le compositeur est un être immergé dans sa culture. Avec les moyens spécifiques de la musique et sans nécessairement tenter de «raconter quelque chose », il peut vouloir nous proposer en musique des prises de position qu'il revient à l'exégèse historique et culturelle d'interpréter.

Mais si les emprunts à la narratologie demeurent largement métaphoriques, il convient peut-être de se demander, en terminant, pourquoi la musicologie

22 «It will be literature that will appear as the basic formative force affecting alike his music, his criticism and his aesthetic theory». 
est tentée, à ce moment-ci, de traiter la musique comme un récit. Dans un ouvrage consacré au soutien de l'Etat à la création musicale contemporaine, un brillant sociologue français, Pierre-Michel Menger, écrit: «Peut-on expliquer l'obsession du sentiment tonal chez les auditeurs contemporains par analogie avec le goût apparemment indéracinable pour la narration et le récit dans la création littéraire et cinématographique ?» (1983: 280). J'ai discuté ailleurs (Nattiez 1989) ce que je crois être les a priori esthétiques de Menger et je n'y reviendrai pas ici. Mais sa comparaison me paraît significative du climat actuel. Au cours d'un colloque de l'IRCAM (avril 1988), le musicologue belge Célestin Deliège faisait observer que les oeuvres de la période post-webernienne ont pour titre Eclat ou Momente. On ne peut nier, aujourd'hui, que la grande majorité du public n'a pas suivi, mais qu'au contraire, il est séduit par un retour au sens de la continuité que Berio a manifesté très tôt, y compris dans une oeuvre significativement intitulée Chemins, et qui me semble gouverner une oeuvre comme Répons sans que Boulez ne fasse aucune concession à la tonalité ${ }^{23}$. Si je peux risquer une explication d'ordre culturel à la tentation narratologique de la musicologie récente, je dirai qu'en cette période post-moderniste, des musiciens et des musicologues se rejoignent pour tenter de concevoir la musique selon ce qu'elle a de common avec le récit littéraire, selon cette dimension fondamentale de la linéarité dont il faudra alors se demander si elle ne constitue pas, pour l'ensemble des formes symboliques inscrites dans le temps, une constante universelle du plaisir humain.

ABBATE, C.

\section{RÉFÉRENCES}

1990: Unsung Voices: Narrative in XIXth Century Music. Princeton University Press (à paraître).

ADORNO, T.W.

1976: Mahler, une physionomie musicale. Paris: Éditions de Minuit.

BOETTICHER, W.

1941: Robert Schumann: Einführung in Persönlichheit und Werk. Berlin.

CALLAGHAN, J.

1985: «Did Elgar Speak English? Language and National Music Style: Comparative Semiotic Analysis », communication présentée au congrès annuel de l'Australian Musicological Society, Melbourne, sept. 1975, inédit.

CHAILLEY, J.

1971: Carnaval de Schumann. Paris: Alphonse Leduc.

23 Cf. pour une étude spécifique de Répons, de ce point de vue, Nattiez (1987). 
CHATMAN, S.

1978: Story and Discourse: Narrative Structure in Fiction and Film. Ithaca: Cornell University Press.

CONE, E.T.

1974: The Composer's Voice. Berkeley-Los Angeles: University of California Press.

DELATTRE, $P$.

1966: «Les dix intonations de base du français », French Review, XL/1: 1-14.

1967: «La nuance de sens par l'intonation », French Review XLI/3: 326-339.

1969: «L'intonation par les oppositions», Le français moderne, décembre 1969: 6-13.

FRANCÈS, R.

1958: La perception de la musique. Paris: Vrin.

GEORGIADES, T.

1954: Musik und Sprache. Heidelberg: Springer Verlag.

GRABOCZ, M.

1987: «Liszt. La Sonate en si mineur: une stratégie narrative complexe», Analyse musicale, $\mathrm{n}^{\circ}$ 8: 64-70.

HALL, R.H.

1953: «Elgar and Intonation of British English», Gramophon, vol. XXXI, $\mathrm{n}^{\circ}$ 6: 6-7.

IMBERTY, M.

1979: Entendre la musique. Paris: Dunod.

1981: Les écritures du temps. Paris: Dunod.

\section{LEVI-STRAUSS, $\mathrm{C}$.}

1971: L'homme nu. Paris: Plon.

\section{LIPPMAN, E. A.}

1964: "Theory and Practice in Schumann's Aesthetics», Journal of the American Musicological Society, vol. XVII: 310-345.

MENGER, P.M.

1983: Le paradoxe du musicien. Paris: Flammarion.

MOLINO, J.

1975: «Fait musical et sémiologie de la musique», Musique en Jeu, no 17: $37-62$.

MOLINO, J., TAMINE, J.

1987: Introduction à l'analyse de la poésie, vol. I. Paris: Presses Universitaires de France. 
NATTIEZ, J. J.

1973: «Y a-t-il une diégèse musicale ?», in P. Faltin - H. P. Reinecke ed., Musik und Verstehen, Köhn, Arno Volk Verlag - Hans Gerig KG: 247-257.

1975: Fondements d'une sémiologie de la musique. Paris: 10-18, Union Générale d'Editions.

1987: Musicologie générale et sémiologie. Paris: Christian Bourgois éditeur.

1989: «Le paradoxe du sociologue (Esthétique et perception dans les travaux de P. M. Menger)», Contrechamps, $\mathrm{n}^{\circ}$ 10: 140-167.

NETTL, B.

1956: «Some Linguistic Approaches to Musical Analysis», Journal of the International Folk Music Council, vol. X: 37-41.

NEWCOMB, A.

1984: «Once More Between Absolute and Program Music: Schumann's Second Symphony », 19th Century Music, Vol. VII, n $3:$ : 233-250.

1987: «Schumann and Late Eighteenth-Century Narrative Strategies», 19th Century Music, vol. XI, $\mathrm{n}^{\circ}$ 2: 164-174.

1989: "Stratégies narratives et perception de la musique du début du dixneuvième siècle ", Contrechamps, $\mathrm{n}^{\circ}$ 10: 12-24.

NORTON, R.

1984: Tonality in Western Culture. University Park: Pennsylvania State University Press.

PROPP, V.

1970: Morphologie du conte. Paris: Gallimard.

RICOEUR, P.

1971: "The Model of the Text: Meaningful Action Considered as a Text ", Social Research, vol. XXXVIII, ${ }^{\circ}$ 3: 529-562. Original français paru in Du texte à l'action (Essais d'herméneutique, II), Paris, Seuil, 1986: 183-211.

1983: Temps et récit, tome I. Paris: Seuil.

TARASTI, E.

1984: «Pour une narratologie de Chopin», International Review of Aesthetics and Sociology of Music, Vol. XV, $\mathrm{n}^{\circ} 1$.

1984: «Une analyse sémiotique: la mise en évidence d'un parcours narratif», Analyse musicale, $\mathrm{n}^{\circ}$ 16: 67-74.

TREITLER, L.

1982: «To Worship that Celestial Sound, Motives for Analysis», The Journal of Musicology, vol. 1: 153-170.

VEYNE, P.

1971: Comment on écrit l'histoire. Paris: Seuil. 